\title{
Influence of Gradient and Smoothness of Atrial Wall Thickness on Initiation and Maintenance of Atrial Fibrillation
}

\author{
Luca Azzolin ${ }^{1}$, Giorgio Luongo ${ }^{1}$, Sara Rocher Ventura ${ }^{2}$, Javier Saiz ${ }^{2}$, Olaf Dössel ${ }^{1}$, Axel Loewe ${ }^{1}$ \\ ${ }^{1}$ Institute of Biomedical Engineering, Karlsruhe Institute of Technology, Karlsruhe, Germany \\ ${ }^{2}$ Ci2B, Universitat Politècnica de València, Valencia, Spain
}

\begin{abstract}
This work uses a highly detailed computational model of human atria to investigate the effect of spatial gradient and smoothing of atrial wall thickness on inducibility and maintenance of atrial fibrillation (AF) episodes. An atrial model with homogeneous thickness $(\mathrm{HO})$ was used as baseline for the generation of different atrial models including either a low (LG) or high thickness gradient between left/right atrial free wall and the other regions. Since the model with high spatial gradient presented non-natural sharp edges between regions, either 1 (HG1) or 2 (HG2) Laplacian smoothing iterations were applied. Arrhythmic episodes were initiated using a rapid pacing protocol and long-living rotors were detected and tracked over time. Thresholds optimised with receiver operating characteristic analysis were used to define high gradient/curvature regions. Greater spatial gradients increased the atrial model inducibility and unveiled additional regions vulnerable to maintain AF drivers. In the models with heterogeneous wall thickness (LG, HG2 and HG1), $73.5 \pm 8.7 \%$ of the long living rotors were found in areas within $1.5 \mathrm{~mm}$ from nodes with high thickness gradient, and $85.0 \pm 3.4 \%$ in areas around high endocardial curvature. These findings promote wall thickness gradient and endocardial curvature as measures of AF vulnerability.
\end{abstract}

\section{Introduction}

Atrial fibrillation (AF) is a frequent and progressive heart condition correlated with high morbidity and mortality. The lack of basic understanding of underlying atrial dysfunctional and structural substrates may be the cause of the low success rate in treatment of persistent AF patients. Computational heart modelling could aid the development of quantitative tools to evaluate the influence of structural features on AF vulnerability.

Recent theoretical studies have highlighted the effect of surface curvature [1] and tissue gradient [2] on the dynamics of re-entrant drivers (RDs). The anchoring of scroll waves to regions with heterogeneous atrial wall thickness have been proven both experimentally and computationally [3]. However, simplified models were used and no detailed atrial model have been exploited to assess correlation between these structural features and regions vulnerable to initiate and maintain AF.

We used a detailed 3-dimensional computational model of human atria including anatomical wall thickness. We varied the spatial gradient between adjacent regions and the degree of smoothing at transitions.

We hypothesized that heterogeneous thickness influences both initiation and maintenance of $\mathrm{AF}$ episodes. Moreover, we sought to investigate the drift of meandering scroll waves along regions of large spatial gradients in wall thickness.

\section{Methods}

\subsection{Atrial models generation}

In this work, a highly detailed volumetric bi-atrial geometry derived from magnetic resonance images was used [4]. The volumetric mesh had an average edge length of $0.4 \mathrm{~mm}$ and a homogeneous wall thickness (HO) of $3 \mathrm{~mm}$. This model was used as baseline for the generation of 3 model variants varying the regional myocardial thickness and the smoothness between the different regions of the atria. The heterogeneous myocardial thickness was introduced following the method proposed in [5] consisting in moving the endocardial nodes in the normal direction until the desired Euclidean distance between the endocardial node and the closest node on the epicardium is reached. The regional thickness ranges were taken from [6-8] and are shown in Fig. 1. The blue stars mark the final regional thickness value for the model with low gradient (LG) between the left/right atrial wall (LAW)/(RAW) and the other regions. The thickness values in the model with a high gradient between LAW/RAW and the other regions are indicated by red stars. Moreover, since the later model entailed unrealistically sharp edges at the transition between regions with highly different thickness, we ap- 
plied 1 iteration (HG1) or 2 iterations (HG2) of Laplacian smoothing to the endocardial surface.

Fiber orientation was calculated by a semi-automatic rule-based algorithm [9]. A variant of the Courtemanche et al. model reflecting atrial fibrillation-induced remodeling was used as ionic model [10]. Nine regions with different anisotropy ratio were implemented to consider the heterogeneity in the atria [11]. Conductivities of all models were tuned to reach a last sinus rhythm activation time of $160 \mathrm{~ms}$ and a global longitudinal conduction velocity of $0.7 \mathrm{~m} / \mathrm{s}$. The spreading of the electrical wave along the myocardium was simulated by solving the monodomain system using openCARP/CARP [12].
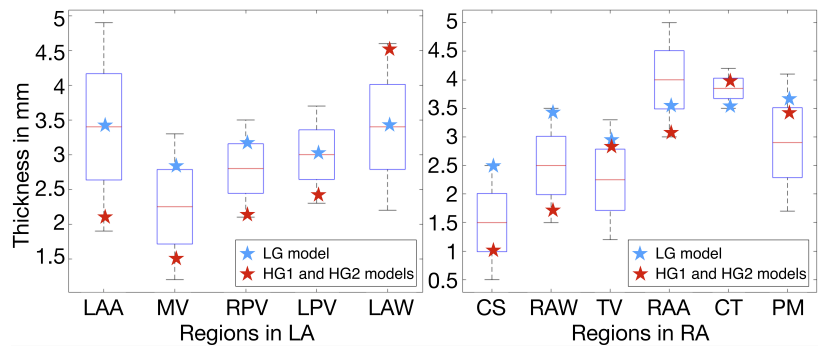

Figure 1. Regional thickness for the models LG, HG1 and HG2 (left atrial appendage=LAA, mitral valve $=M V$, right pulmonary veins $=R P V$, left pulmonary veins $=\mathrm{LPV}$, coronary sinus $=\mathrm{CS}$, tricuspid valve $=\mathrm{TV}$, right atrial appendage $=\mathrm{RAA}$, crista terminalis $=\mathrm{CT}$, pectinate muscles=PM).

\subsection{Thickness gradient and endocardial curvature}

To better identify the cause of scroll wave drifting, we computed two thickness features: thickness gradient and endocardial curvature. The curvature of the endocardial surface at each node was calculated as $1 / R$, where $R$ is the radius of the fitted osculating sphere.

\subsection{Protocol to test inducibility}

We tested inducibility of arrhythmic episodes by pacing from 48 evenly distributed points on the endocardial surface with an inter-point distance of $2 \mathrm{~cm}$. The rapid pacing protocol consisted in a train of pulses with decreasing coupling interval from $160 \mathrm{~ms}$ to $110 \mathrm{~ms}$. We considered a point to be 'inducing' if by pacing from that location, an arrhythmia was induced and sustained for at least $1.5 \mathrm{~s}$ after the last beat of the pacing protocol.

\subsection{Detection and tracking of AF dynam- ics}

Reentrant waves have an organising center in many cases. In 3D this is known as filament, which represents the center-line of a scroll wave. We followed the method presented in [13] to detect filaments and we tracked the spatio-temporal behavior of each filament along the whole episode duration. We defined long-living rotors as filaments sustained for at least $500 \mathrm{~ms}$.

\subsection{Optimal threshold for high thickness gradient and endocardial curvature}

An iterative binary classification (filaments present vs. not present) was computed to create receiver operating characteristic (ROC) curves. Thickness gradient and endocardial curvature were used as discriminators for the ROC curves. The optimum operating point on the ROC curve was defined as the point on the curve with the shortest distance to the top left corner of the graph and it was selected as threshold.

\section{Results and Discussion}

\subsection{Inducibility}

In model $\mathrm{HO}, 12$ points were 'inducing' (10 lead to macro reentries, 2 to micro reentries). We observed 13 'inducing' points in model LG (10 macro reentries, 3 rotors) and 16 points in model HG1 (6 macro, 10 micro). Furthermore, we identified 16 'inducing' points in model HG2 (11 macro, 5 rotors). Number and location of the 'inducing' points for each model are summarized in Tab.1. The models with higher gradient between LAW/RAW and the other regions (HG1 and HG2) were more vulnerable regarding initiation of reentrant episodes compared with models with more homogeneous thickness (HO and LG). We observed the development of microreentries when pacing precisely in the areas with high thickness gradient. Moreover, the combination of high gradient and sharper edges (model HG1) had the highest number of points inducing rotors.

\subsection{Maintenance}

In total, 4 long-living rotors were sustained in average for $978.8 \mathrm{~ms}$ in the model HO regions CT, PMs and RAA. Fig. 2 shows the filaments of these reentries with most of them drifting along the CT or moving in confined areas of the RAA. No micro reentries were maintained in the CS or MV with similar behavior in the model LG. In the model LG, we identified a total of 7 long-living rotors with an average lifespan of $921.7 \mathrm{~ms}$ and most of them were moving 


\begin{tabular}{cccccc}
\hline Model & Macroreentries & \multicolumn{5}{c}{ Microreentries } \\
& & PMs & CT & MV & CS \\
\hline HO & 10 & 2 & & & \\
LG & 10 & 3 & & & \\
HG1 & 6 & 5 & 1 & 3 & 1 \\
HG2 & 11 & 2 & 2 & 1 & \\
\hline
\end{tabular}

Table 1. Number of point inducing reentrant arrhythmic episodes in each model classified in macro reentry and micro reentry. The points inducing micro reentries are further divided per location.

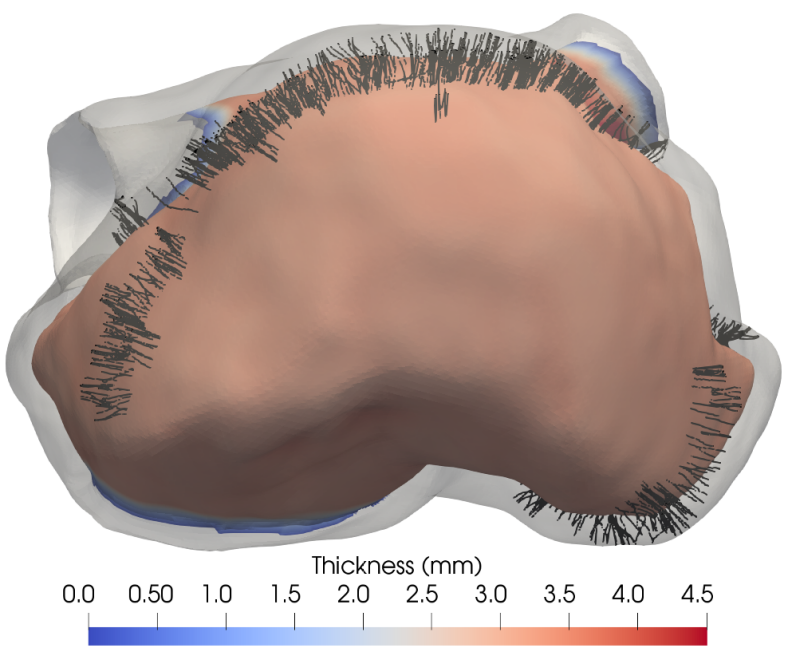

Figure 2. Filaments of long-living rotors in the right atrium of model HO (black lines). The endocardial surface is colored according by wall thickness. Pectinate muscles view.

along the PMs. In the models HG1 and HG2, we identified more reentrant wave meandering following the PMs or the MV and, finally, being maintained close to the steps between RAW and CS, CT or RAA, as shown in Fig. 3. We found a total of 11 and 16 long-living rotors maintaining in average for $1021.5 \mathrm{~ms}$ and $982.5 \mathrm{~ms}$ in the models HG2 and HG1, respectively. Nodes in which a long-living rotor was found (see Fig. 4), mostly corresponded to locations with high gradient magnitude and surface curvature (see Fig. 4). We detected 70.5\%/78.7\%/71.3\% of filaments in areas within $1.5 \mathrm{~mm}$ from the regions with high thickness gradient and $81.1 \% / 87.1 \% / 86.9 \%$ of filaments in areas within $1.5 \mathrm{~mm}$ from the regions with high endocardial curvature, in the models LG, HG2 and HG1 respectively. The optimal thresholds extracted from the ROC curves were $0.1 / 0.2 / 0.46 \mathrm{~mm} / \mathrm{mm}$ for the gradient magnitude and $0.7 / 0.8 / 0.96 \mathrm{~mm}^{-1}$ for the curvature, for the models LO, HG2 and HG1, respectively. In our simulations, no fibrotic tissue was included to clearly analyse the effect of anatomical thickness features on reentry dynamics.

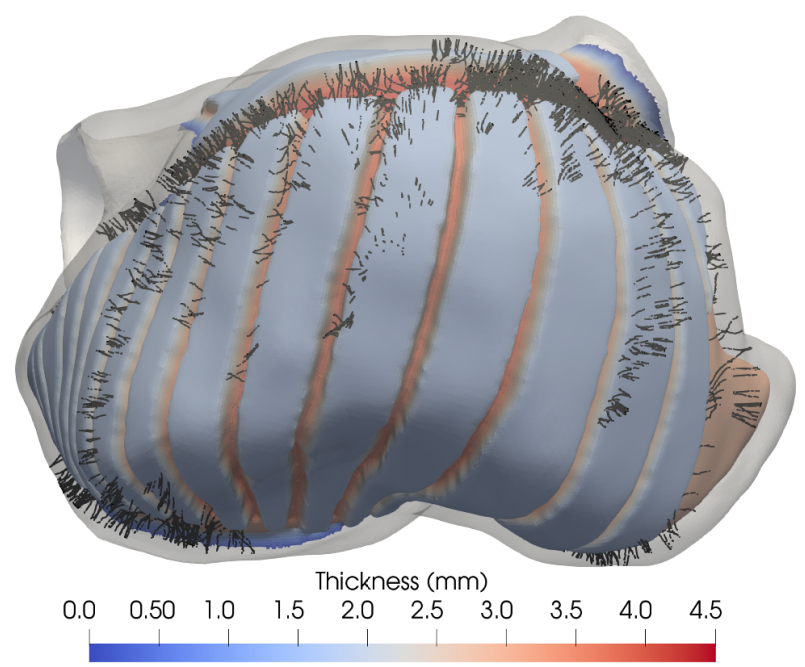

Figure 3. Filaments of long-living rotors in the right atrium of model HG2 (black lines). The endocardial surface is colored according by wall thickness. Pectinate muscles view.

\section{Conclusion}

In this work, we showed that the gradient and the degree of smoothing of anatomical thickness influence both the vulnerability to initiate and to maintain atrial fibrillation episodes. We presented how thickness heterogeneity increased not only the number of pacing location from which it was possible to induce an arrhythmia but also the complexity of the arrhythmia, developing more micro reentries. The inclusion of higher gradient highlighted pathways followed by meandering rotors (PMs), as shown experimentally [14] and unveiled new areas vulnerable to sustain drivers (CS and MV). Furthermore, we showed how rotors are attracted by steep edges and are drifting to the border between regions with different thickness, as theoretically predicted in [2]. Furthermore, we noticed that the degree of smoothing affected inducibility, increasing the number of points initiating rotors, and sustainability, leading to a higher number of long-living drivers. We conclude that wall thickness gradient and curvature could be valuable a priori measures to assess AF vulnerability and predict maintenance areas to ablate.

\section{Acknowledgments}

Research supported by the European Union's Horizon 2020 research and innovation programme under the Marie Skłodowska-Curie grant agreement No.766082 (MY-ATRIA project). 


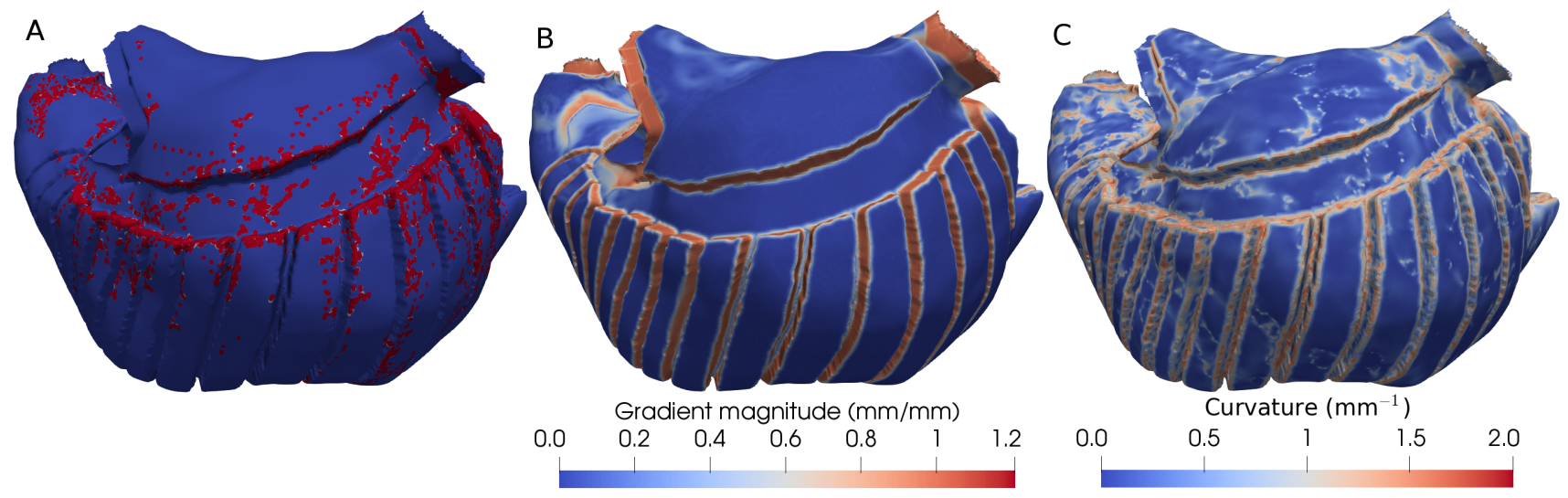

Figure 4. Model HG1: A) endocardial nodes in which a long-living rotor was encountered are shown in red; B) thickness gradient magnitude; C) endocardial curvature showed as inverse of the osculating sphere radius. Crista terminalis and pectinate muscles view.

\section{References}

[1] Dierckx H, Brisard E, Verschelde H, Panfilov AV. Drift laws for spiral waves on curved anisotropic surfaces. Phys Rev E 2013;88:012908.

[2] Biktasheva IV, Dierckx H, Biktashev VN. Drift of scroll waves in thin layers caused by thickness features: Asymptotic theory and numerical simulations. Phys Rev Lett 2015; 114:068302.

[3] Yamazaki M, Mironov S, Taravant C, Brec J, Vaquero LM, Bandaru K, Avula UM, Honjo H, Kodama I, Berenfeld O, Kalifa J. Heterogeneous atrial wall thickness and stretch promote scroll waves anchoring during atrial fibrillation. Cardiovasc res 2012;94(1):48-57.

[4] Krueger MW, Seemann G, Rhode K, Keller DUJ, Schilling C, Arujuna A, Gill J, O’Neill MD, Razavi R, Dössel O. Personalization of atrial anatomy and electrophysiology as a basis for clinical modeling of radio-frequency ablation of atrial fibrillation. IEEE Trans Med Imaging 2013;32(1):7384.

[5] Rocher-Ventura S, Martínez-Mateu L, López-Pérez AD, Ferrer Albero A, Sáncez-Quintana D, Saiz Rodríguez FJ. A three-dimensional model of the human atria with heterogeneous wall thickness and fibre transmurallity - a realistic platform for the study of atrial fibrillation. CinC 2019;1-4.

[6] Suenari K, Nakano Y, Hirai Y, Hiroshi O, Noboru O, Yuko M, Shigeyuk U, Kenta K, Takehito T, Chikaaki M, Mai F, Kazuaki C, Yasuki K. Left atrial thickness under the catheter ablation lines in patients with paroxysmal atrial fibrillation: insights from 64-slice multidetector computed tomography. Heart and Vessels 2013;28:360-368.

[7] Varela M, Morgan R, Theron A, Dillon-Murphy D, Chubb H, Whitaker J, Henningsson M, Aljabar P, Schaeffter T, Kolbitsch C, Aslanidi OV. Novel mri technique enables non-invasive measurement of atrial wall thickness. IEEE Trans Med Imaging 2017;36(8):1607-1614.

[8] Wi J, Lee H, Uhm J, Kim J, Pak H, Lee M, Kim YJ, Joung B. Complex fractionated atrial electrograms related to left atrial wall thickness. J Cardiovasc Electrophysiol 2014; 25(11):1141-1149.

[9] Wachter A, Loewe L, Krueger MW, Dössel O, Seemann G. Mesh structure-independent modeling of patient-specific atrial fiber orientation. Curr Dir in Biomed Eng 2015; 1:409-412.

[10] Loewe A, Wilhelms M, Dössel O, Seemann G. Influence of chronic atrial fibrillation induced remodeling in a computational electrophysiological model. Biomed Eng 2014; 59(S1):S929-S932.

[11] Loewe A, Krueger MW, Platonov PG, Holmqvist F, Dössel $\mathrm{O}$, Seemann G. Left and right atrial contribution to the Pwave in realistic computational models. Lect Notes Comput Sci 2015;9126:439-447.

[12] Vigmond EJ, Hughes M, Plank G, Leon LJ. Computational tools for modeling electrical activity in cardiac tissue. J Electrocard 2003;36:69-74.

[13] Clayton R, Zhuvhkova E, Panfilov AV. Phase singularities and filaments: Simplifying complexity in computational models of ventricular fibrillation. Prog Biophys Mol Biol 2006;90:378-398.

[14] Hansen BJ, Zhao J, Csepe TA, Moore BT, Li N, Jayne LA, Kalyanasundaram A, Lim P, Bratasz A, Powell KA, Simonetti OP, Higgins RS, Kilic A, Mohler PJ, Janssen PM, Weiss R, Hummel JD, Fedorov VV. Atrial fibrillation driven by micro-anatomic intramural re-entry revealed by simultaneous sub-epicardial and sub-endocardial optical mapping in explanted human hearts. Eur Heart J 2015;36(35):23902401.

Address for correspondence:

Luca Azzolin

Fritz-Haber-Weg 1, 76131 Karlsruhe, Germany

publications@ibt.kit.edu 